مجلة العلوم الهندسية_العدد الثاني-2006

\title{
Theoretical Performance Comparison between Inline, Offset and Twin Crankshaft Internal Combustion Engine Models
}

\author{
Dr.Uzaldin S. Abdulhussain*", Dr.Taj Elssir Hassan ${ }^{* *}$ and Maisara Mohy \\ Eldin Gasim*
}

\begin{abstract}
Twin crankshaft is a new engine arrangement introduced to overcome cylinder's liner wear problems encountered in the conventional inline crankshaft engine due to the effect of the side thrust force. The offset crankshaft arrangement was also introduced to solve the same problem.

In this work a computer programs was built to obtain the theoretical performance comparison between the three engines arrangements (inline, twin and offset crankshaft engines), and compared the theatrical performance with the experimental results, which done to the engine's models.

The study results show that the twin crankshaft engine model exhibited no thrust force, and that the thrust force in the offset crankshaft model is smaller than that in the inline crankshaft engine model. These agree with experimental results obtained from the same engine model.
\end{abstract}

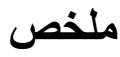

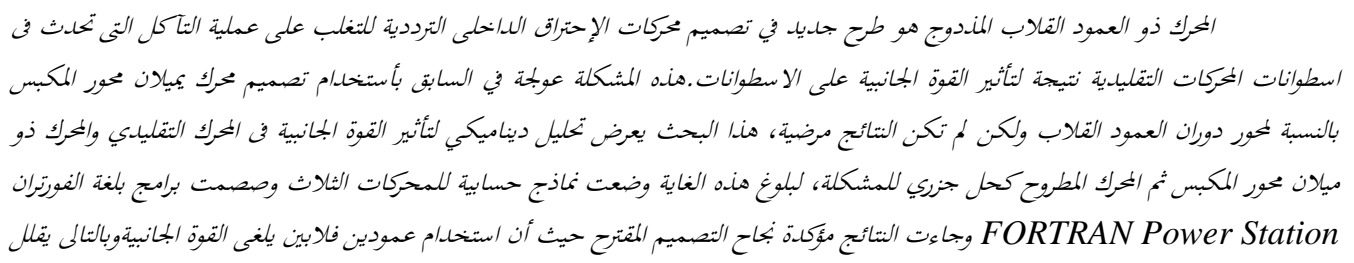

عملية التآل .

\footnotetext{
* Mechanical Engineering Dept., Sudan University for Science and Technology.

** Mechanical Engineering Dept., Omdurman Islamic University.

*** Mechanical Engineering Dept., Elimam Elmahdi University.
} 


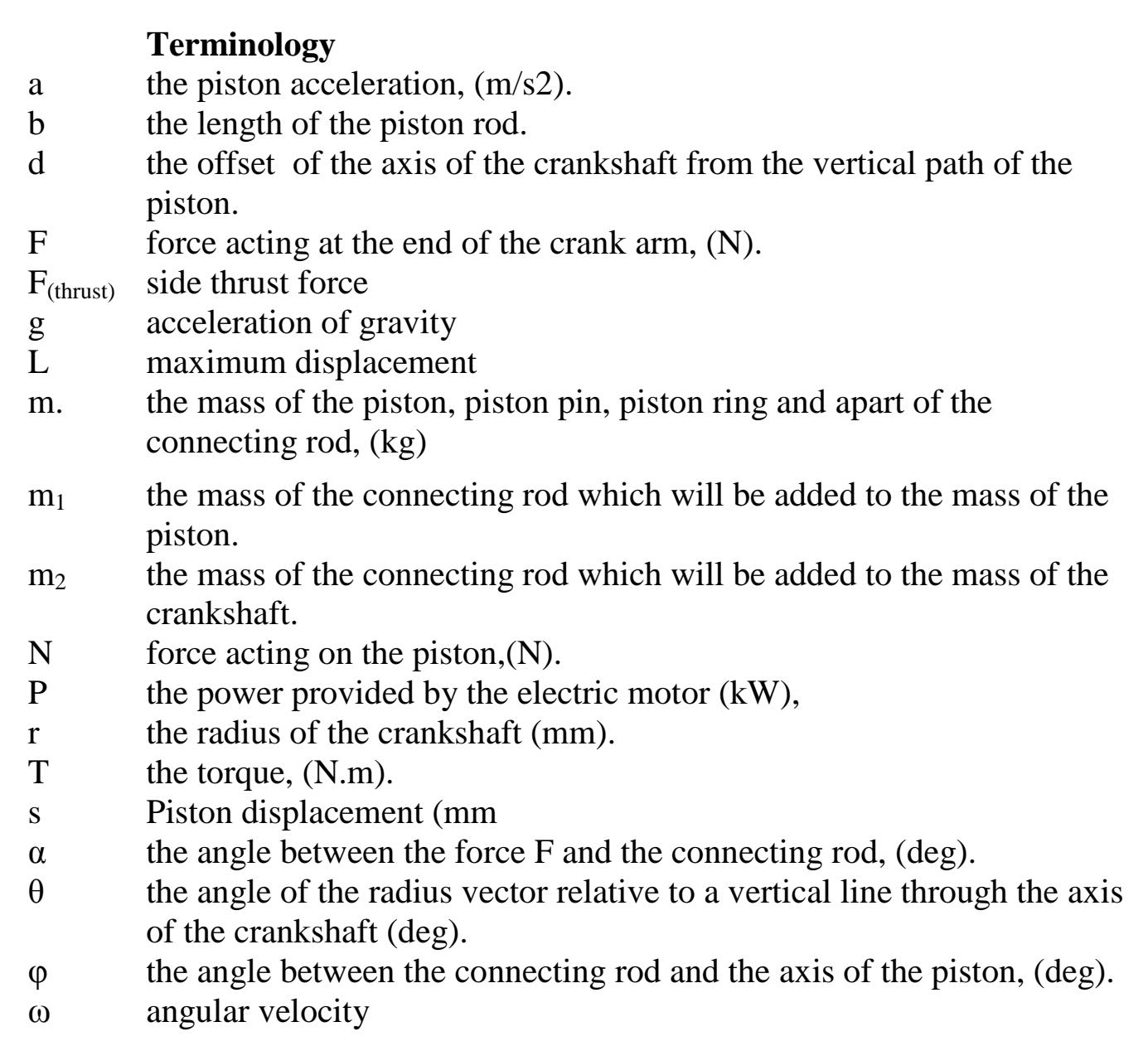

\section{Introduction}

The main factor causing wear in the cylinder's liner is the side thrust force [1], which is created because the connecting rod makes an angle with the axis of the piston so that as the piston pushed down, it pushed it self to one side, hard against the cylinder wall. The side thrust force switches sides as the piston passes through top and bottom center.

When using twin crankshaft engine, fitted with two connecting rods, every connecting rod has its own side thrust force and, as a result, they cancel each other [2, 3]. 
By simply moving the crankshaft a bit to one side, the connecting rod can be made more upright during the combustion stroke so that the force of the piston against the cylinder wall is smaller and hence friction is reduced, and that is what happens when using an offset crankshaft [4].

Besides canceling the side thrust force, the twin crankshaft engine has many other advantages, just like reducing engine's vibrations and noise, and maintaining excellent engine's balance.

When using two crankshafts with two connecting rods attached to a single piston, that means the rotation point of the crankshafts must be offset from the axis of the piston movement.

This offset makes differences in piston displacement, speed and acceleration from the conventional engines. Also the intake and power strokes are not equal to pressure and exhaust strokes [5], and the angle between the connecting rod and the axis of the piston $(\varphi)$ is grater than the conventional ones.

The length of crank arm (r), connecting rod (b) and the offset of the crank from the ordinary point (d), controls the offset and twin crankshafts engines specifications.

To make a comparison between the inline (conventional) engine and the offset crankshaft engine and the twin crank shaft engine a FORTRAN program used to calculate the three engines performance. The program first calculate the displacement, speed and acceleration of the offset crankshaft engine with different values of (r), (b) and (d). Then the program used to calculate the side thrust force, acceleration and the force $(\mathrm{N})$ applying on the piston for the three engines (inline, offset and twin crankshaft) supposing that an electric motor drives the engines.

The initial conditions for the program was similarly to a model of a twin crankshaft engine built in Sudan University of science and technology/ Faculty of Engineering. The same mode after taking off one of its crankshafts was converted to an offset crankshaft engine model, and by transferring the point of the crankshaft rotation to the axis of the piston movement the model becomes an inline (conventional) crankshaft engine model. The model of the three engines arrangement was driven by an electric motor. 


\section{The Computer Program}

As mentioned above the crank offset changes the values of piston displacement, speed and acceleration. The first part of the computer program deals with this issue. The second part deals with the inline crankshaft engine model performance, the third part with the offset crankshaft engine model and the last part deals with the twin crankshaft engine model.

\subsection{First part: piston displacement, speed and acceleration with offset crankshaft}

To derive the new equations we represent the rotation of the crank as a circle where the center of the circle would be the axis of the crankshaft and the circumference represents the rotation of the center of the journal. The piston's connecting rod is then represented as a straight line. Since the piston and the top of the piston rod travel the same vertical velocity, the piston itself is not a factor. Since this article is about the offset crankshaft, notice that the center of the circle representing the rotation of the journal is offset from the vertical line, which denotes the center of the piston [6]. Figure 1 shows this representation.

From figure (1):

$\mathrm{Y}=\mathrm{Y}_{1}+\mathrm{Y}_{2}$

$\mathrm{Y}_{2}=\mathrm{r}^{*} \cos \theta$

$\mathrm{X}_{1}=\mathrm{r} * \sin \theta$

$\mathrm{Y}_{1}^{2}=\mathrm{b}^{2}-\left(\mathrm{X}_{1}+\mathrm{X}_{2}\right)^{2}$

$\mathrm{X}_{2}=\mathrm{d}$

From (1): $Y=Y_{1}+Y_{2}$

From (2): $Y=r^{*} \cos \theta+Y_{1}$

From (4): $Y=r^{*} \cos \theta+\sqrt{ }\left\{b^{2}-\left(X_{1}+X_{2}\right)^{2}\right\}$

From (3): $Y=r^{*} \cos \theta+\sqrt{ }\left\{b^{2}-\left(r * \sin \theta+X_{2}\right)^{2}\right\}$

$\mathrm{S}(\theta)=1-\left[\mathrm{r}^{*} \cos \theta+\sqrt{ }\left\{\mathrm{b}^{2}-(\mathrm{r} * \sin \theta+\mathrm{d})^{2}\right\}\right]$

But: $\theta=\omega t$ 


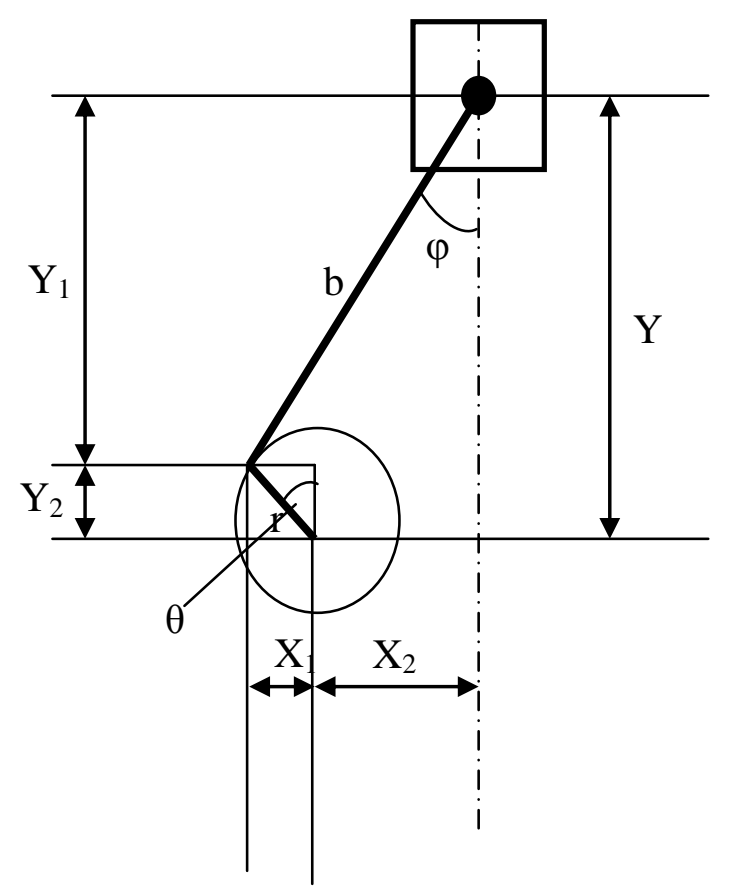

Figure (1): Engine with Offset

Crankshaft

Then:

$\mathrm{S}(\omega \mathrm{t})=1-\left[\mathrm{r}^{*} \cos (\omega \mathrm{t})+\sqrt{ }\left\{\mathrm{b}^{2}-\left(\mathrm{r}^{*} \sin (\omega \mathrm{t})+\mathrm{d}\right)^{2}\right\}\right]$

Taking the first and second derivative of the displacement to obtain the equations for velocity and acceleration respectively.

$$
\begin{aligned}
& s(\omega t)=l-\left[r \cos \omega t+{\sqrt{b^{2}-(r \sin \omega t+d)}}^{2}\right] \\
& v(\omega t)=r \omega \sin \omega t+\frac{2 \omega(r \sin \omega t+d) r \cos \omega t}{\sqrt{b^{2}-(r \sin \omega t+d)}} \\
& a(\omega t)=r \omega^{2} \cos \omega t+\frac{(r \sin \omega t+d)^{2} r^{2} \omega^{2} \cos ^{2} \omega t}{\left[\sqrt{b^{2}-(r \sin \omega t+d)^{2}}\right]^{3}}-\frac{(r \sin \omega t+d) r \omega^{2} \sin \omega t+r^{2} \omega^{2} \cos ^{2} \omega t}{\sqrt{b^{2}-(r \sin \omega t+d)^{2}}}
\end{aligned}
$$


Equations (6), (7) and (8) give the values of displacement, speed and acceleration with offset crankshaft. These equations used to built a FORTRAN program, which calculate the following values with different values of $(r),(b)$ and $(\mathrm{d})$ :

1. Piston displacement. 2. Piston speed. 3. Piston acceleration. 4. The maximum value of angle $(\varphi) .5$. The (intake-power) stroke. 6. The (exhaustpressure) stroke.

To calculate the value of the angle $(\varphi)$, from Figure 1:

$$
\varphi=\tan ^{-1}[(d+r \sin (\theta)) / b]
$$

To calculate the strokes in degrees of the crank angle, we find the angle of the crank at maximum piston displacement (i.e. top dead center TDC), and the angle at minimum displacement (i.e. bottom dead center BDC).

Figure 2 shows the program flow chart.

\subsubsection{FORTRAN Program For Calculating Piston Displacement, Speed, Acceleration and the Angle $\varphi$, with an Offset Crankshaft Engine:}

program acceleration

integer $\mathrm{i}$

$\mathrm{i}=0$

omega $=261$

$\mathrm{r}=0.02$

do while $(\mathrm{r}<0.03)$

$\mathrm{d}=0$

do while $(\mathrm{d}<0.03)$

$\mathrm{b}=0.08$

do while $(b<0.1)$

do $1 \mathrm{I}=0,361$

theta $=i * 3.142857143 / 180.0$

$\mathrm{s}=\mathrm{r} * \cos ($ theta $)+\operatorname{sqrt}((\mathrm{b} * * 2)-(\mathrm{r} * \sin ($ theta $)+\mathrm{d}) * * 2)$

$\mathrm{v}=\mathrm{r}^{*} \mathrm{~W} * \sin ($ theta $)+\left(2 *(\mathrm{r} * \sin (\right.$ theta $)+\mathrm{d}) * \mathrm{r}^{*} \mathrm{~W} * * \cos ($ theta $\left.)\right)$

$\& / \operatorname{sqrt}((\mathrm{b} * * 2)-(\mathrm{r} * \sin ($ theta $)+\mathrm{d}) * * 2)$

$\mathrm{a}=-\mathrm{r} * \cos ($ theta $) *(\mathrm{w} * * 2)$

$\mathrm{a} 1=(((\mathrm{r} * \sin ($ theta $)+\mathrm{d}) * * 2) *(\mathrm{r} * * 2) *((\cos ($ theta $)) * * 2) *(\mathrm{w} * * 2))$

$\mathrm{a} 2=(\operatorname{sqrt}((\mathrm{b} * * 2)-(\mathrm{r} * \sin ($ theta $)+\mathrm{d}) * * 2)) * * 3$

$\mathrm{a} 3=(\mathrm{r} * \sin ($ theta $)+\mathrm{d}) * \mathrm{r}^{*} \sin (\mathrm{x}) *(\mathrm{w} * * 2)-(\mathrm{w} * * 2) *\left(\mathrm{r}^{* * 2}\right) *(\cos ($ theta $)) * * 2$

$\mathrm{a} 4=\operatorname{sqrt}((\mathrm{b} * * 2)-(\mathrm{r} * \sin ($ theta $)+\mathrm{d}) * * 2)$

$\mathrm{a} 5=\mathrm{a}-(\mathrm{a} 1 / \mathrm{a} 2)+(\mathrm{a} 3 / \mathrm{a} 4)$ 
phi $=\operatorname{asin}\left(\left(\mathrm{d}+\mathrm{r}^{*} \sin (\mathrm{x})\right) / \mathrm{b}\right)$

write i,s,a5, v,phi

1 continue

$\mathrm{b}=\mathrm{b}+0.01$

end do

$\mathrm{d}=\mathrm{d}+0.01$

end do

$r=r+0.005$

end do

end 


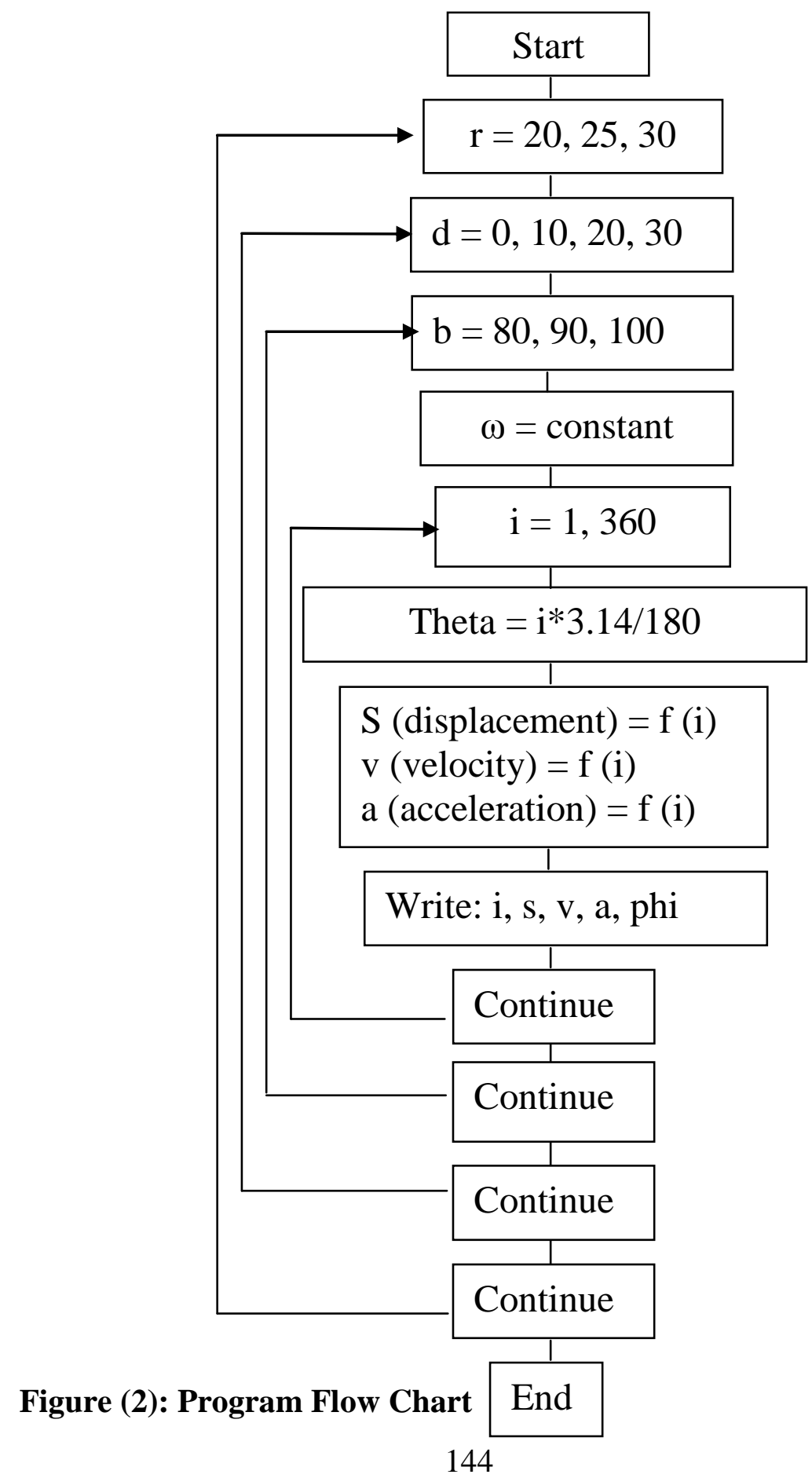


the result after running this program are shown in Table (3).

\subsection{Part Two: Inline Crankshaft Engine Performance}

The main equations used to calculate the force applying on the piston, acceleration and the side thrust force could be calculated as following:

Forces balance:

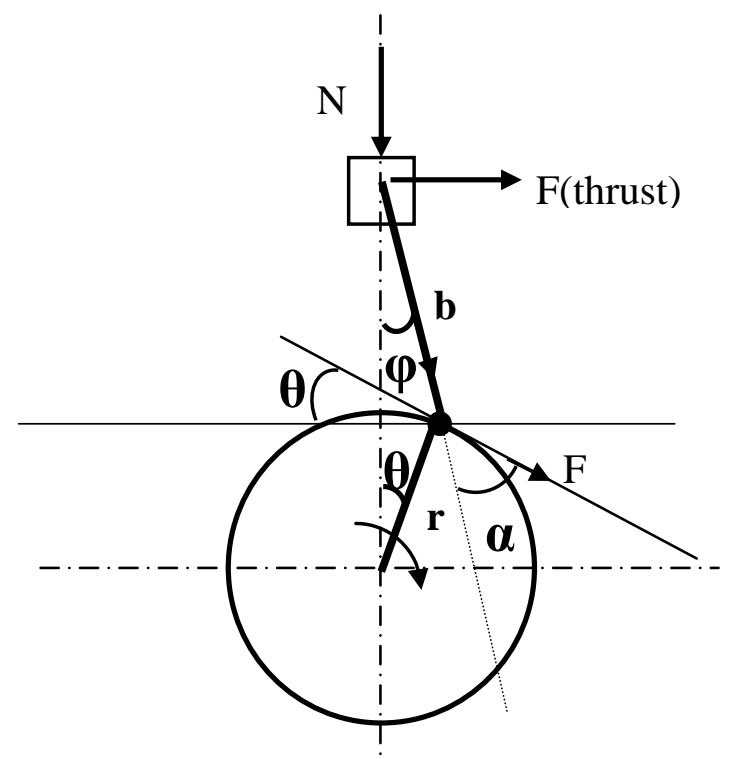

Figure (3): Inline Crankshaft

Engine

From figure (3):

$\mathrm{N}=\left[\mathrm{F}^{*} \cos (\alpha) / \cos (\varphi)\right]-\mathrm{m}^{*} \mathrm{a} \pm \mathrm{m}^{*} \mathrm{~g}$

$\mathrm{F}=\mathrm{P} / \mathrm{T}=\mathrm{P} / \mathrm{r}^{*} \omega=\mathrm{P} /(2 \pi \mathrm{n} / 60)^{*} \mathrm{r}(\mathrm{N})$

$\operatorname{Sin}(\varphi)=r^{*} \sin (\theta) / b \Rightarrow \varphi=\sin ^{-1}\left(r^{*} \sin (\theta) / b\right)$

$\theta+\varphi+\alpha=90^{\circ}$

$\Rightarrow \alpha=90^{\circ}-\left[\theta+\sin ^{-1}\left(\mathrm{r}^{*} \sin (\theta) / \mathrm{b}\right)\right]$

$\mathrm{m}=$ piston + part of the connecting rod $(\mathrm{kg})$

$\mathrm{a}=\mathrm{r}^{*} \omega^{2} *[\cos (\theta)+\cos (2 * \theta) /(2 * \mathrm{~b} / \mathrm{r})]$

$\mathrm{N}=\left[\mathrm{F}^{*} \cos \left\{90^{\circ}-\left[\theta+\sin ^{-1}\left(\mathrm{r}^{*} \sin (\theta) / \mathrm{b}\right)\right]\right\}^{*} \cos \left\{\sin ^{-1}\left(\mathrm{r}^{*} \sin (\theta) / \mathrm{b}\right)\right\}\right]-$

$\mathrm{m} * \mathrm{a} \pm \mathrm{m} * \mathrm{~g} \longrightarrow(9)$ 
$\mathrm{F}_{\text {(thrust) }}=\mathrm{F}^{*} \cos (\alpha)^{*} \sin (\varphi)$

$=\mathrm{F}^{*} \cos \left\{90^{\circ}-\left[\theta+\sin ^{-1}\left(\mathrm{r}^{*} \sin (\theta) / \mathrm{b}\right)\right]\right\}^{*} \mathrm{r}^{*} \sin (\theta) / \mathrm{b}$

The computation requires the masses of the piston and the connecting rod to calculate the moment of inertia. Theoretically the mass of the connecting rod could be divided to two separate masses, one of them added to the piston and it takes the piston acceleration, while the other added to the crankshaft and it takes the crankshaft acceleration.

\subsubsection{Replacing the Mass of the Connecting Rod by Two Separated Masses}

For calculating the moment of inertia for the piston, we must add a part of the connecting rod to the piston, according to the following rules [7]:

$1-\mathrm{m}_{1}+\mathrm{m}_{2}=\mathrm{m}$

2- $\mathrm{m}_{1} \cdot \mathrm{a}=\mathrm{m}_{2} \cdot \mathrm{b}$

Where:

$\mathrm{m}=612.53 \mathrm{~g}$ from table 1.

From equation [1],[2]:

$m_{1}=\frac{b}{a+b} m \longrightarrow[\mathrm{a}]$
$m_{2}=\frac{a}{a+b} m \longrightarrow[\mathrm{b}]$

From equation [a] and figure (4):

$\mathrm{m}_{1}=0.0612 * 0.61253 / 0.160=0.2343 \mathrm{~kg}$

From equation[a]:

$\mathrm{m}_{2}=0.0988^{*} 0.61253 / 0.160=0.3782 \mathrm{~kg}$ 


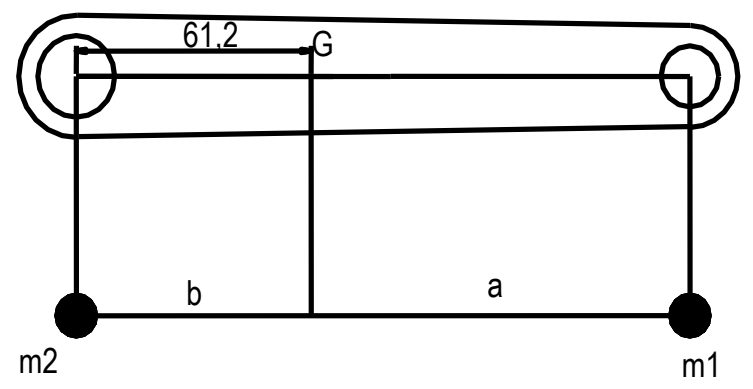

Figure (4): Center of Gravity for Connecting Rod 1

Remember that the model was for a twin crankshaft engine, that means it has two connecting rods, but when we use the model as an inline crankshaft engine, we toke off one of two crankshafts with its connecting rod. So by using the same method for the other connecting rod, and figure 5:

$\mathrm{m}_{1}=0.061253 * 0.61045 / 0.16=0.2451 \mathrm{~kg}$

$\mathrm{m}_{2}=0.09597 * 0.61045 / 0.16=0.3674 \mathrm{~kg}$

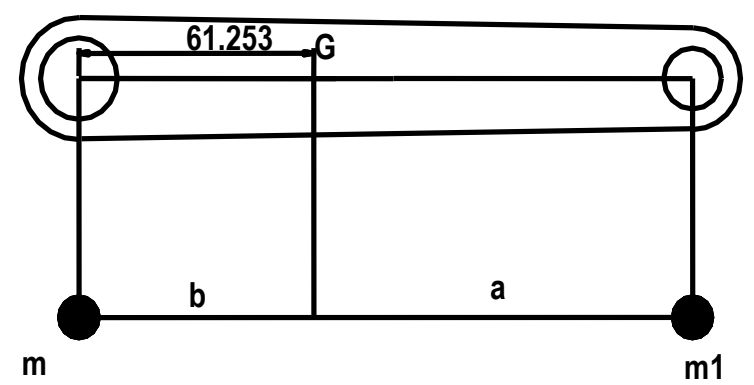

Figure (5): Center of Gravity for Connecting Rod 2 
Table (1): Model Components Weight

\begin{tabular}{|l|l|}
\hline \multicolumn{1}{|c|}{ Item } & Weight $(\mathrm{kg})$ \\
\hline Piston \&piston rings & 0.20681 \\
\hline Connecting rod 1 & 0.61253 \\
\hline Connecting rod 2 & 0.61045 \\
\hline
\end{tabular}

By using equations (9) and (10) a FORTRAN program built to calculate the force applying on the piston and the side thrust force. Figure 6 shows the program flow chart.

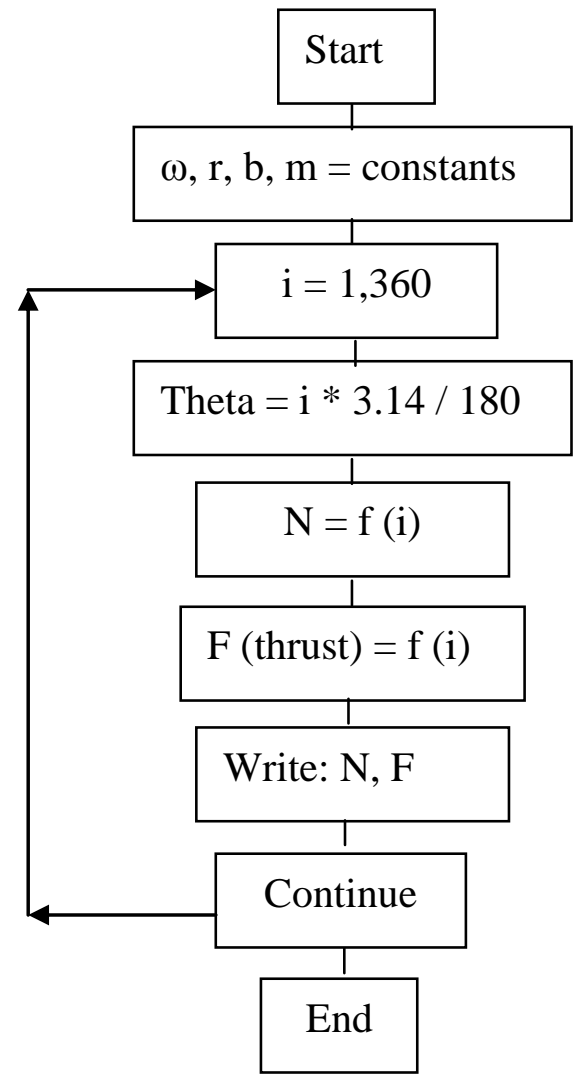

Figure (6): Program Flow Chart for the Inline Crankshaft Engine 


\subsubsection{FORTRAN program used to get the inline engine performance:}

program inlinecrankshaft engine

integer $\mathrm{i}$

omega $=$ constant

$\mathrm{r}=0.023$

$\mathrm{b}=0.092$

$\mathrm{m}=0.441$

pi $=3.141592654$

do $10 \mathrm{I}=0,360$

theta $=\mathrm{i}^{*} \mathrm{pi} / 180$

$\mathrm{F}=196.6^{*} \cos ((\mathrm{pi} / 2)-$

$\left(\right.$ theta $+\operatorname{asin}\left(\left(\mathrm{r}^{*} \sin (\right.\right.$ theta $\left.\left.\left.\left.)\right) / \mathrm{b}\right)\right)\right) * \cos (\operatorname{asin}((\mathrm{r} * \sin ($ theta $)) / \mathrm{b}))$

$\mathrm{a}=\mathrm{r} *(\mathrm{w} * * 2) *(\cos ($ theta $)+(\cos (2 *$ theta $)) /(2 *(\mathrm{~b} / \mathrm{r})))$

if(i.le.180) $\mathrm{N}=\mathrm{F}-\mathrm{a} * \mathrm{~m}+\mathrm{m} * 9.81$

if(i.gt.180) $\mathrm{N}=\mathrm{F}-\mathrm{a} * \mathrm{~m}-\mathrm{m} * 9.81$

Fthrust $=196.6 * \cos ((\mathrm{pi} / 2)-$

$($ theta $\operatorname{asin}((\mathrm{r} * \sin ($ theta $)) / \mathrm{b}))) *((\mathrm{r} * \sin ($ theta $)) / \mathrm{b})$

Write N, Fthrust, a

10 continue

end

the results obtained after running the program are shown in table (4).

\subsection{Part Three: Offset Crankshaft Engine Performance}

The offset crankshaft is an engine introduced to reduce the effect of the side thrust force, which responsible of wear occurs on the liner, and the vibrations and noise problems.

Force balance: 


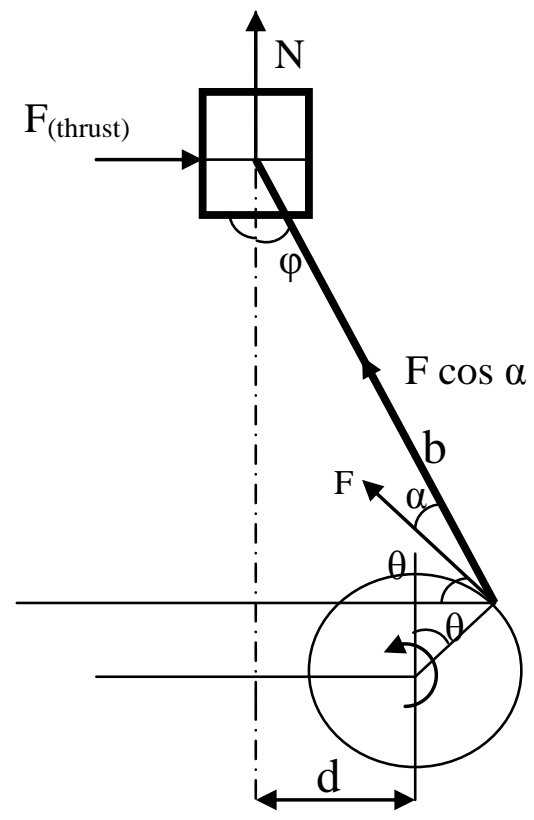

Figure (7): Offset Crankshaft Engine

Figure (7) shows the force balance for the offset crankshaft engine. From figure (7):

$\mathrm{N}=\left[\mathrm{F}^{*} \cos \alpha^{*} \cos \varphi\right]-\mathrm{m}^{*} \mathrm{a} \pm \mathrm{mg}$.

Force $(\mathrm{F})$ calculations:

$\mathrm{P}=\mathrm{T}^{*} \omega=\mathrm{F}^{*} \mathrm{r}^{*} \omega \Rightarrow \mathrm{F}=\mathrm{P} / \mathrm{r}^{*} \omega$

$\omega=2 \pi \mathrm{n} / 60$

$\varphi=\sin ^{-1}[(d+r \sin \theta) / b]$

And: $\varphi+\theta+\alpha=90^{\circ}$. Then: $\alpha=90-(\varphi+\theta)=90-\left(\sin ^{-1}[(d+r \sin \theta) / b]+\theta\right)$

Substituting these values in equation $[\mathrm{c}]$, then:

$\mathrm{N}=\left[\mathrm{F}^{*} \cos \left\{90-\left(\sin ^{-1}[(\mathrm{~d}+\mathrm{r} \sin \theta) / \mathrm{b}]+\theta\right)\right\}^{*} \cos \left\{\sin ^{-1}[(\mathrm{~d}+\mathrm{r} \sin \theta) / \mathrm{b}]\right\}\right]-\mathrm{m}^{*} \mathrm{a}$ $\pm \mathrm{mg} \longrightarrow(11)$

$\left.\mathrm{F}_{\text {(thrust) }}=\left[\mathrm{F}^{*} \cos \left\{90-\left(\sin ^{-1}[(\mathrm{~d}+\mathrm{r} \sin \theta) / \mathrm{b}]+\theta\right)\right\}^{*}[(\mathrm{~d}+\mathrm{r} \sin \theta) / \mathrm{b}]\right\}\right]$ (12)

To calculate the force applying on the piston, the acceleration and the side thrust force, equations (11) and (12) used in a FORTRAN program with program flow chart similar to one shown in Figure (6). 


\subsubsection{FORTRAN Program to Calculate the Offset Crankshaft Engine Performance}

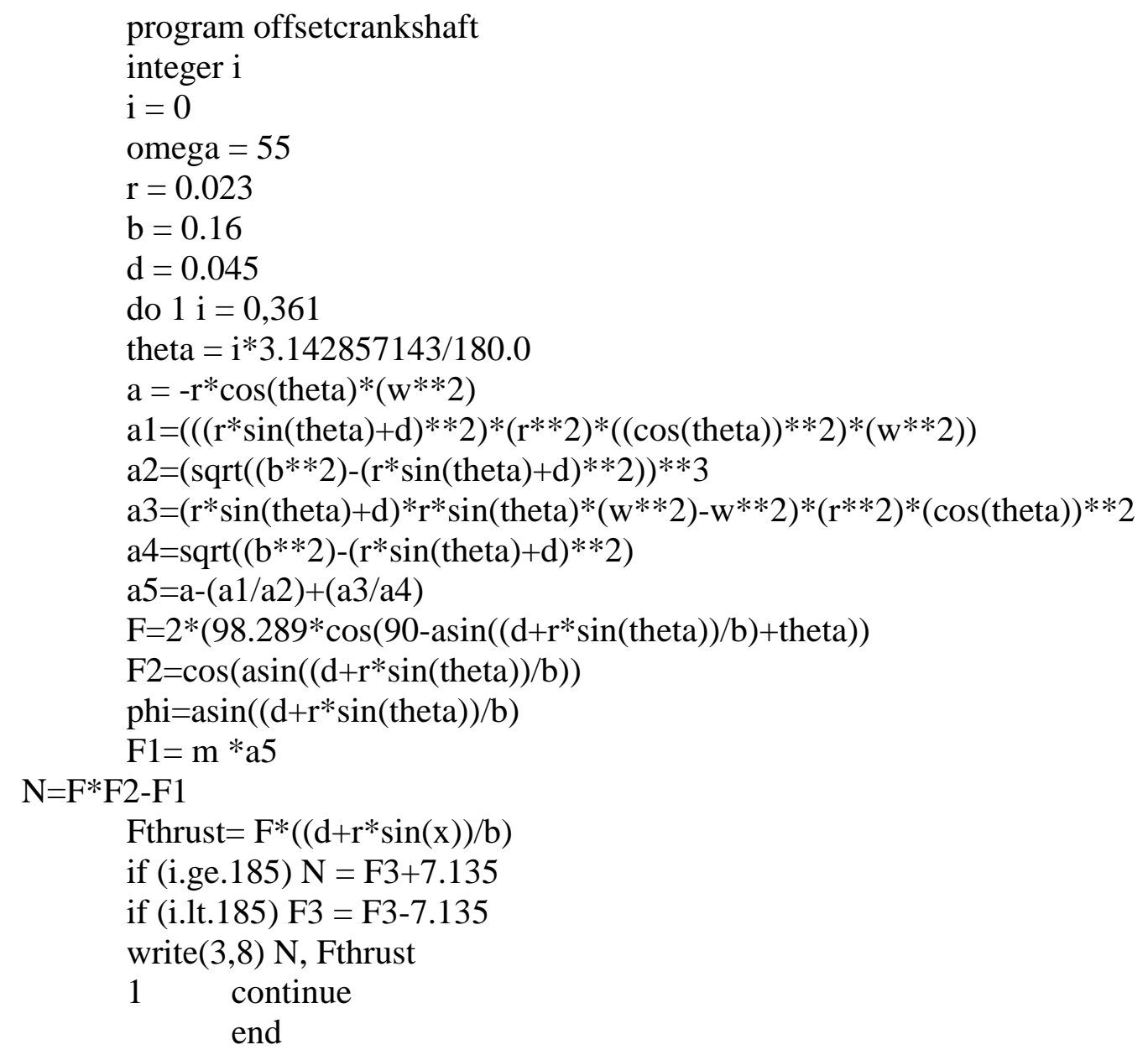

The results obtained after running the program are shown in table (5).

\subsection{Part Four: Twin Crankshaft Engine Performance}

The twin crankshaft engine is consisting of two crankshafts, tow flanges, two connecting rods, a piston and liner. Figure 8 shows the twin crankshaft model components. 


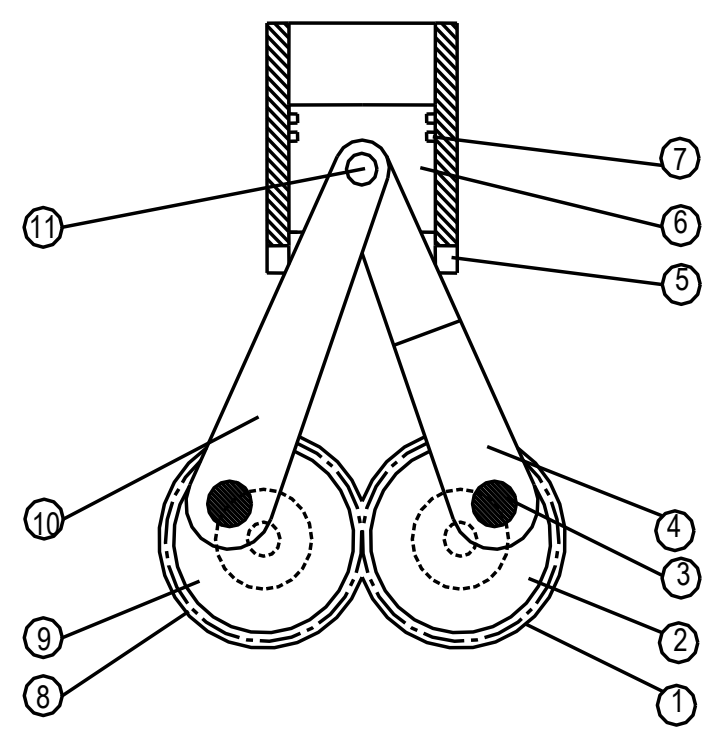

$\begin{array}{llll}1 & 1^{\text {st }} \text { gear } & 7 & \text { Piston's rings } \\ 2 & 1 \text { st flange } & 8 & 2^{\text {nd }} \text { gear } \\ 3 & \text { Crank pin } & 9 & 2^{\text {nd }} \text { flange } \\ 4 & 1^{\text {st }} \text { connecting } & 10 & 2^{\text {nd }} \text { connecting } \\ & \text { rod } & & \text { rod } \\ 5 & \text { Liner } & 11 & \text { Piston wrist pin }\end{array}$

Figure (8): Twin Crankshaft Engine Components

Using Figure (9), which shows the force balance in the twin crankshaft engine, the theoretical values for the force applying on the piston and the side thrust force were calculated.

From Figure (9):

$\mathrm{N}=\left[\mathrm{F}^{*} \cos \alpha^{*} \cos \varphi\right]-\mathrm{m} * \mathrm{a} \pm \mathrm{mg}$.

Force $(\mathrm{F})$ calculations:

$\mathrm{P}=\mathrm{T}^{*} \omega=\mathrm{F}^{*} \mathrm{r}^{*} \omega=\mathrm{F}=\mathrm{P} / \mathrm{r}^{*} \omega$

$\omega=2 \pi \mathrm{n} / 60$

$\mathrm{N}=\left[\mathrm{F}^{*} \cos \alpha^{*} \cos \varphi\right]-0.0 .68621 * \mathrm{a} \pm 0.72733 * 9.81$. 
But from figure (5):

$\varphi=\sin ^{-1}[(d+r \sin \theta) / b]$

And:

$\varphi+\theta+\alpha=90^{\circ}$. Then: $\alpha=90-(\varphi+\theta)=90-\left(\sin ^{-1}[(d+r \sin \theta) / b]+\theta\right)$

Substituting these values in equation (c), then:

$\Rightarrow \mathrm{N}=2 *\left[\mathrm{~F}^{*} \cos \left\{90-\left(\sin ^{-1}[(\mathrm{~d}+\mathrm{r} \sin \theta) / \mathrm{b}]+\theta\right)\right\}^{*} \cos \left\{\sin ^{-1}[(\mathrm{~d}+\mathrm{r} \sin \theta) / \mathrm{b}]\right\}\right]$

$-\mathrm{m} * \mathrm{a} \pm \mathrm{mg}$

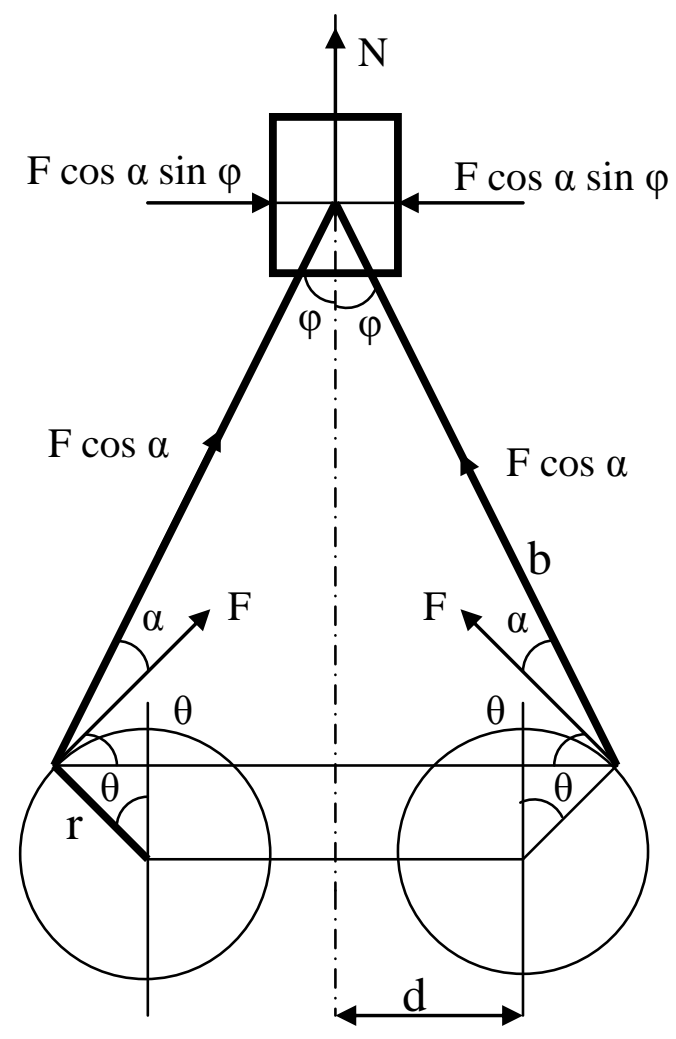

Figure (9): twin crankshaft engine piston's forces balance

as shown from Figure 9, there is no side thrust force in the twin crank shaft engine, that means:

$\mathrm{F}$ thrust $=0 \longrightarrow(14)$ 
A FORTRAN program was written to calculate the acceleration, and the force applying on the piston, for a crank angle from $0^{\circ}$ to $360^{\circ}$. The program flow chart is similar to that shown in Figure (6).

\subsubsection{FORTRAN Program to Calculate the Twin Crankshaft Engine Performance}

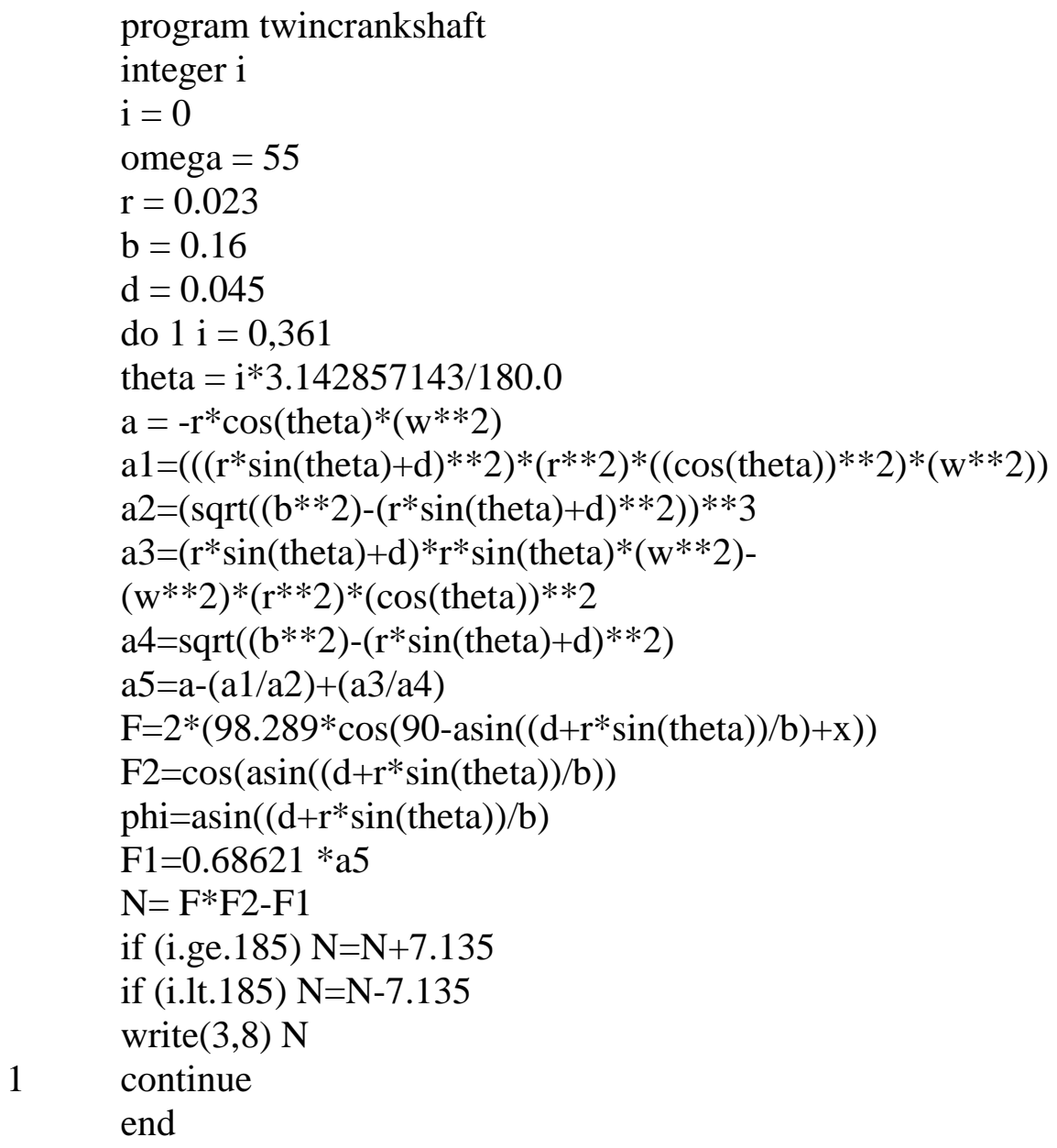

\section{Results and Discussions}

Using equations $(6,7,8,9,10,11,12,13,14)$, a Fortran program was written to calculate the acceleration, side thrust force and the force applying 
on the piston, and the displacement, speed and strokes of the engines, for a crank angle from $0^{\circ}$ to $360^{\circ}$.

Tables 2,3,4,5 and figures 10,11, 12 and 13 show the results.

Table (2): Inline Crankshaft Engine Acceleration, Force Applying on the Piston and the Side Thrust Force:

\begin{tabular}{|c|c|c|c|}
\hline $\begin{array}{c}\text { Crank } \\
\text { angle } \\
(\mathrm{deg})\end{array}$ & $\begin{array}{c}\text { Acceleration } \\
\left(\mathrm{m} / \mathrm{s}^{2}\right)\end{array}$ & Force $(\mathrm{N})$ & $\begin{array}{c}\text { Side thrust } \\
\text { force }(\mathrm{N})\end{array}$ \\
\hline 0 & 69.64687 & 26.39399 & $0.00 \mathrm{E}+00$ \\
\hline 20 & 65.43082 & -51.63659 & 3.750969 \\
\hline 40 & 53.29742 & -119.9942 & 12.91964 \\
\hline 60 & 34.72611 & -168.787 & 22.56177 \\
\hline 80 & 11.9755 & -193.5434 & 27.81737 \\
\hline 100 & -12.1972 & -194.5941 & 26.43666 \\
\hline 120 & -34.8741 & -175.0811 & 19.48433 \\
\hline 140 & -53.3289 & -139.1347 & 10.31472 \\
\hline 160 & -65.3507 & -90.97118 & 2.837276 \\
\hline 180 & -69.5031 & $-3.48 \mathrm{E}+01$ & $3.87 \mathrm{E}-05$ \\
\hline 200 & -65.2907 & 33.75497 & 2.877342 \\
\hline 220 & -53.2161 & 92.49712 & 10.37952 \\
\hline 240 & -34.722 & 144.6551 & 19.54749 \\
\hline 260 & -12.024 & 184.0577 & 26.46976 \\
\hline 280 & 12.14887 & 204.1696 & 27.80252 \\
\hline 300 & 34.87872 & 199.2878 & 22.50297 \\
\hline 320 & 53.41074 & 166.6793 & 12.84483 \\
\hline 340 & 65.49104 & 108.8672 & 3.699465 \\
\hline
\end{tabular}


Table (3): The Displacement, Maximum Piston Speed, Acceleration, Angle $(\varphi)$ and the Intake and Pressure Strokes with Different Values of Crank Arm (r), Offset (d), and the Length of the Connecting Rod (b):

\begin{tabular}{|c|c|c|c|c|c|c|c|c|}
\hline $\begin{array}{c}\mathrm{r} \\
(\mathrm{mm})\end{array}$ & $\begin{array}{c}\mathrm{d} \\
(\mathrm{mm})\end{array}$ & $\begin{array}{c}\mathrm{b} \\
(\mathrm{mm})\end{array}$ & $\begin{array}{l}\text { Displacement } \\
\text { (m) }\end{array}$ & $\begin{array}{l}\text { Maximum } \\
\text { Speed, } \\
(\mathrm{m} / \mathrm{s})\end{array}$ & $\begin{array}{c}\text { Maximum } \\
\text { Acceleration } \\
\left(\mathrm{m} / \mathrm{s}^{2}\right)\end{array}$ & $\begin{array}{c}\text { Maximum } \\
\varphi \\
\text { (Degrees) }\end{array}$ & $\begin{array}{c}\text { Intake } \\
\text { stroke } \\
\text { (Degrees) }\end{array}$ & $\begin{array}{l}\text { Pressure } \\
\text { stroke } \\
\text { (Degrees) }\end{array}$ \\
\hline \multirow{12}{*}{20} & \multirow{3}{*}{0} & 80 & .040000 & 5.230601 & 1021.815 & 14.48485 & 180 & 180 \\
\hline & & 90 & .040000 & 5.229271 & 1059.66 & 12.84610 & 180 & 180 \\
\hline & & 100 & .040000 & 5.228226 & 1089.936 & 11.54281 & 180 & 180 \\
\hline & \multirow{3}{*}{10} & 80 & ".040338 & 5.237028 & 1078.475 & 22.03548 & 184 & 176 \\
\hline & & 90 & .040262 & 5.234789 & 1090.590 & 19.48109 & 183 & 177 \\
\hline & & 100 & .040210 & 5.233074 & 1108.642 & 17.46645 & 182 & 178 \\
\hline & \multirow{3}{*}{20} & 80 & .041410 & 5.244868 & 1194.918 & 30.01521 & 188 & 172 \\
\hline & & 90 & .041084 & 5.241053 & 1167.907 & 26.40110 & 186 & 174 \\
\hline & & 100 & .040861 & 5.238430 & 1161.325 & 23.59013 & 185 & 175 \\
\hline & \multirow{3}{*}{30} & 80 & .043432 & 5.657467 & 1387.399 & 31.51738 & 192 & 168 \\
\hline & & 90 & .042584 & 5.249264 & 1294.357 & 33.76610 & 189 & 171 \\
\hline & & 100 & .042027 & 5.244871 & 1249.223 & 30.01521 & 187 & 173 \\
\hline \multirow{12}{*}{25} & \multirow{3}{*}{0} & 80 & .050000 & 6.542137 & 1198.103 & 18.21919 & 180 & 180 \\
\hline & & 90 & .050000 & 6.539951 & 1232.466 & 16.13580 & 180 & 180 \\
\hline & & 100 & .050000 & 6.538251 & 1277.269 & 14.48485 & 180 & 180 \\
\hline & \multirow{3}{*}{10} & 80 & .050438 & 6.550787 & 1333.175 & 25.95764 & 185 & 175 \\
\hline & & 90 & .050338 & 6.547251 & 1319.939 & 22.89698 & 184 & 176 \\
\hline & & 100 & .050268 & 6.544589 & 1327.311 & 20.49770 & 183 & 177 \\
\hline & \multirow{3}{*}{20} & 80 & ".051842 & 6.562311 & 1538.667 & 34.24622 & 190 & 170 \\
\hline & & 90 & .051401 & 6.556084 & 1461.077 & 30.01521 & 188 & 172 \\
\hline & & 100 & .051104 & 6.551742 & 1426.492 & 26.75724 & 187 & 173 \\
\hline & \multirow{3}{*}{30} & 80 & .054525 & 7.366423 & 1871.620 & 43.45456 & 196 & 164 \\
\hline & & 90 & .053354 & 6.679641 & 1675.406 & 37.68898 & 193 & 167 \\
\hline & & 100 & .052608 & 6.560997 & 1575.341 & 33.38393 & 190 & 170 \\
\hline \multirow{12}{*}{30} & \multirow{3}{*}{0} & 80 & ".060000 & 7.855540 & 1423.229 & 22.03548 & 180 & 180 \\
\hline & & 90 & .060000 & 7.852183 & 1425.471 & 19.48109 & 180 & 180 \\
\hline & & 100 & .060000 & 7.849609 & 1449.296 & 17.46645 & 180 & 180 \\
\hline & \multirow{3}{*}{10} & 80 & .060553 & 7.867294 & 1644.735 & 30.01521 & 187 & 173 \\
\hline & & 90 & .060421 & 7.861577 & 1584.384 & 26.40118 & 185 & 175 \\
\hline & & 100 & .060331 & 7.857644 & 1562.566 & 23.59013 & 183 & 177 \\
\hline & \multirow{3}{*}{20} & 80 & .062339 & 7.883499 & 1971.952 & 38.70180 & 193 & 167 \\
\hline & & 90 & .061752 & 7.873888 & 1809.129 & 33.76610 & 190 & 170 \\
\hline & & 100 & .061370 & 7.867299 & 1724.858 & 30.01521 & 188 & 172 \\
\hline & \multirow{3}{*}{30} & 80 & .065830 & 9.215178 & 2526.240 & 48.61502 & 201 & 159 \\
\hline & & 90 & .064227 & 8.323761 & 2147.836 & 41.83151 & 195 & 165 \\
\hline & & 100 & .063243 & 7.879830 & 1956.695 & 36.88859 & 192 & 168 \\
\hline
\end{tabular}


Table (4): Offset Crankshaft Engine Acceleration, Force Applying on the Piston and the Side Thrust Force:

\begin{tabular}{|r|r|r|r|}
\hline $\begin{array}{l}\text { Crank } \\
\text { angle } \\
(\mathrm{deg})\end{array}$ & \multicolumn{1}{l|}{$\begin{array}{l}\text { Acceleration } \\
(\mathrm{N})\end{array}$} & Force $(\mathrm{N})$ & $\begin{array}{l}\text { Side thrust } \\
\text { force }(\mathrm{N})\end{array}$ \\
\hline 0 & 80.89231 & 6.326322 & $-9.87 \mathrm{E} 00$ \\
\hline 20 & 67.54444 & -51.07028 & -29.8254 \\
\hline 40 & 42.6129 & -105.1166 & -51.6636 \\
\hline 60 & 11.27526 & -150.3619 & -70.8842 \\
\hline 80 & -19.5274 & -180.5339 & -82.2356 \\
\hline 100 & -43.6843 & -189.4166 & -81.4010 \\
\hline 120 & -58.2845 & -172.5222 & -67.0826 \\
\hline 140 & -63.9644 & -129.1493 & -42.388 \\
\hline 160 & -63.1957 & -64.02534 & -14.1626 \\
\hline 180 & -58.2338 & $1.26 \mathrm{E}+01$ & $9.94 \mathrm{E}+00$ \\
\hline 200 & -50.0658 & 78.55049 & 25.02212 \\
\hline 220 & -38.4392 & 138.4455 & 30.68857 \\
\hline 240 & -22.5405 & 175.4288 & 30.08532 \\
\hline 260 & -1.98668 & 187.2101 & 27.13418 \\
\hline 280 & 22.19965 & 176.2005 & 24.09378 \\
\hline 300 & 47.09035 & 147.0719 & 20.7539 \\
\hline 320 & 68.20893 & 104.7379 & 15.20888 \\
\hline 340 & 80.69968 & 53.5164 & 5.315868 \\
\hline
\end{tabular}

Table (5): Twin Crankshaft Engine Acceleration, Force Applying on the Piston:

\begin{tabular}{|l|l|l|}
\hline $\begin{array}{l}\text { Crank angle } \\
(\mathrm{deg})\end{array}$ & $\begin{array}{l}\text { Acceleration } \\
(\mathrm{N})\end{array}$ & Force $(\mathrm{N})$ \\
\hline 0 & 80.89231 & 28.55853 \\
\hline 20 & 67.54444 & -32.1098 \\
\hline 40 & 42.6129 & -92.2671 \\
\hline 60 & 11.27526 & -145.194 \\
\hline 80 & -19.5274 & -182.916 \\
\hline 100 & -43.6843 & -197.719 \\
\hline 120 & -58.2845 & -184.404 \\
\hline 140 & -63.9644 & -142.423 \\
\hline 160 & -63.1957 & -77.1106 \\
\hline 180 & -58.2338 & $7.25 \mathrm{E}-01$ \\
\hline
\end{tabular}

157 


\begin{tabular}{|l|l|l|}
\hline 200 & -50.0658 & 63.87416 \\
\hline 220 & -38.4392 & 126.619 \\
\hline 240 & -22.5405 & 167.4992 \\
\hline 260 & -1.98668 & 184.3185 \\
\hline 280 & 22.19965 & 179.2372 \\
\hline 300 & 47.09035 & 156.2095 \\
\hline 320 & 68.20893 & 119.0519 \\
\hline 340 & 80.69968 & 70.892 \\
\hline 360 & 80.84415 & 14.66689 \\
\hline
\end{tabular}

3.1 Table (3) shows the variation of piston displacement, speed, acceleration, angle $(\varphi)$ and the (intake-power), (exhaust-pressure) strokes, with three values for the crank arm (r), for values for the offset value (d), and four values for the length of the connecting rod (b). When $d=0$ the engine returns to a conventional one, with four equal strokes, and the piston displacement equals 2r. But when an offset happens, the strokes becomes unequal, the intake-power strokes are greater than the two other strokes.

This phenomena gives a grate advantage to the offset and twin crankshaft engines, because when the intake stroke becomes greater than $180^{\circ}$, that gives the air an addition time to fill the cylinder, and that improves the volumetric efficiency.

Also when the power stroke becomes greater than $180^{\circ}$, that maintained a complete, clean burn, which improves the engine's efficiency $[2,3]$.

3.2 Figure (10) shows the theoretical acceleration comparison between the twin crankshaft engine, the inline crankshaft engine and the offset crankshaft engine. It shows that there is no a sensitive difference in the acceleration value between the three engines arrangements, but the maximum and minimum values of the acceleration in the case of twin and offset engines are located in different crank angle compared with the inline crankshaft engine. The offset is the chief cause of this behavior.

3.3 Figure (11) shows the theoretical side thrust force comparison between the inline crankshaft engine, the offset crankshaft and the twin crankshaft engines. The side thrust force equals zero for the twin crankshaft engine, and it switching sign after the first $180^{\circ}$ in the offset crankshaft case.

The use of electric motor instead of combustion to drive the model causes the side thrust force in case of inline crankshaft engine model to be always in the 
same side without switching from side to side as expected for the inline crankshaft engine.

These results are agreed with the experimental tests done on the same model. Figure (13) shows a liner wear comparison between the inline, offset and the twin crankshaft engines. The figure shows much wear in the inline crankshaft engine, and less wear in the twin crankshaft engine, because the absence of the side thrust force in the twin crankshaft engine [8].

3.4 Figure (12) shows the theoretical force (applying on the piston) comparison between the twin crankshaft engine, the inline crankshaft engine and the offset crankshaft engine.

The figure shows a similarity between the forces in the three types of engines.

\section{Conclusions}

1. The twin crankshafts decrease the liner wear by canceling the thrust force.

2. The twin crankshaft increases the life of the crankshaft group by distributing the load between the two shafts components.

3. The twin crankshafts offer a good possibility of fixing weight balances.

4. The twin crankshafts eliminate engine vibrations produced by the reactive moment.

5. The twin crankshafts increases engine width and entail the use of more moving parts of the engine. This point should economically be considered. 
-

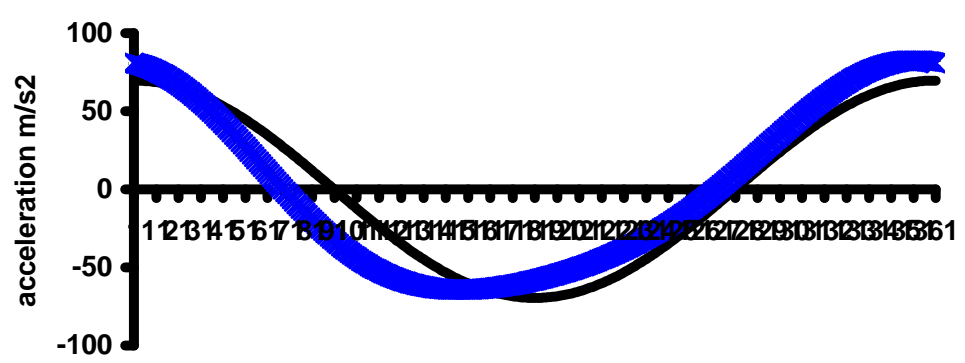

crank angle (deg)

Figure (10): Piston Acceleration Comparison between Inline, Offset and Twin Crankshaft Engines

$$
\text { inline }
$$

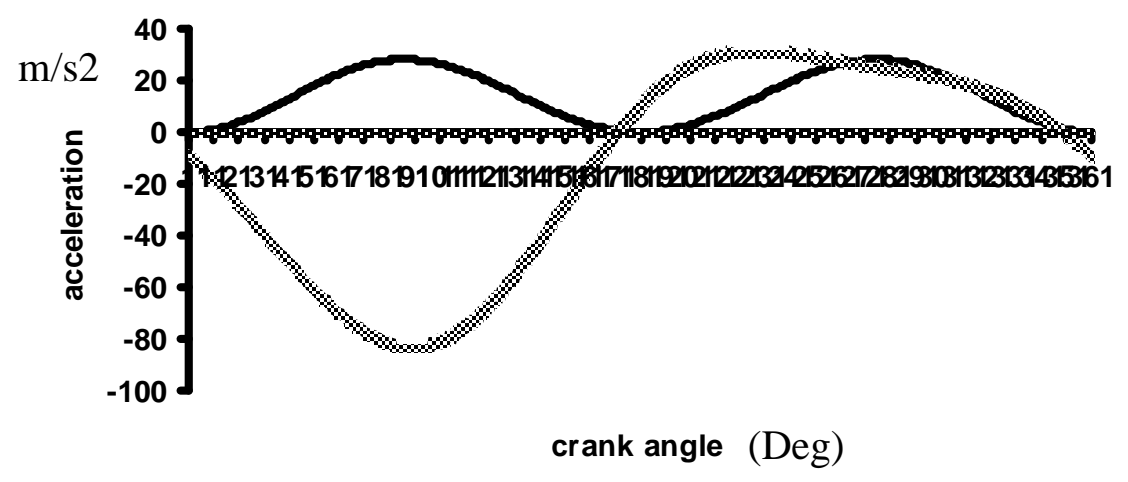

Figure (11): Side Thrust Force Comparison between Inline, Offset and Twin Crankshaft Engines 


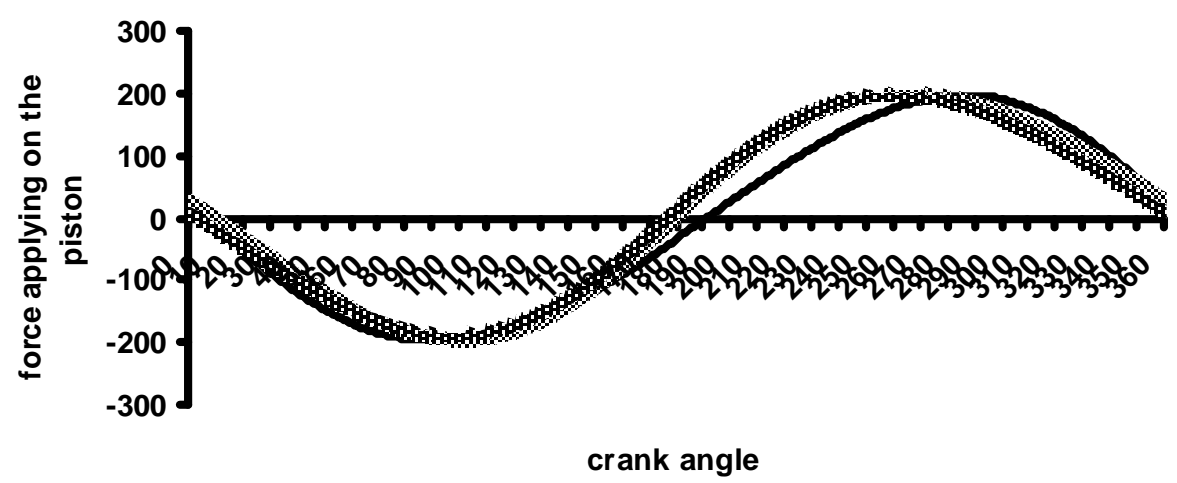

Figure (12): Piston Force Comparison between Inline, Offset and Twin Crankshaft Engines

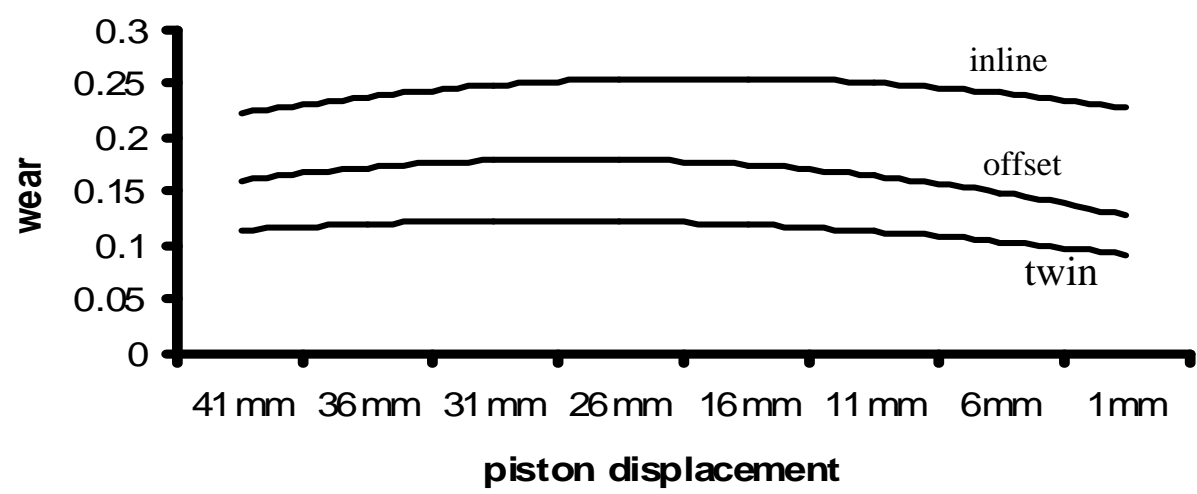

Figure (13): Liner Wear Comparison between the Inline, Offset and Twin Crankshaft Engines 


\section{References}

[1] Colin R. Ferguson, Internal Combustion Engines-Applied Thermosciences, John Wiley \& Sons, 1986.

[2] Feuling; James J, Contra-rotating twin crankshaft internal combustion engine. U.S paten 5,595.147,

[3] John A. Wittner, Motorcycle With Twin Crankshaft Mechanism, U.S patent 5,823,333, (1998)

[4] Garvin; Edward A., Offset Crankshaft Mechanism for Internal Combustion Engines, U.S. Patent No.58162016, oct. 1998.

[5] Uzaldin S. Abdulhussain, Taj Elssir Hassan and Maisara Mohy Eldin Gasim, Twin Crank Shaft Engine Arrangement ,Sudan Engineering Society JOURNAL, may. 2006, Volume 52 No.46

[6]Mike Rollins, what is the speed of a piston with an offset crankshaft?

[7] J. Hannah \&R.C. Stephens, Mechanics of Machines, Edward Arnold Ltd, 1984.

[8] Uzaldin S. Abdulhussain, Taj Elssir Hassan and Maisara Mohy Eldin Gasim , Cylinder's liner wear comparison between inline, offset and twin crankshaft Internal combustion engines, accepted to publish in Sudan Engineering Society JOURNAL, Sep. 2006, Volume 52 No.47 